\title{
Nota sobre la distribución de Savia sessiliflora (Euphorbiaceae) en México
}

Savia sessiliflora (Sw.) Willd. es una especie no reportada previamente en la literatura para México, aunque había sido recolectada bajo otros nombres por lo menos desde 1939. Lundell (Contr. Univ. Michigan Herb. 7:24, 1942) describió Phyllanthus heterotrichus basado en el ejemplar Kenoyer s. n., 15-VII-1939 (MICH), el cual fue determinado posteriormente como Savia sessiliflora por G.L. Webster en 1954 (agradezco al Dr. W.R. Anderson el haberme proporcionado esta información). Generalmente no fue correctamente determinada sino hasta recientemente, cuando fue identificada primeramente por D. M. Johnson (MICH) en 1982 y por la presente autora en 1983, de la costa del Golfo de México y de la costa del Pacífico respectivamente. Esta especie, antes considerada como un elemento antillano (de las islas del Caribe, en Cuba, Puerto Rico, Islas Vírgenes), ahora se conoce como de amplia distribución en México tanto en selvas bajas caducifolias como en selvas medianas subcaducifolias, desde Tamaulipas, San Luis Potosí y Jalisco hasta Veracruz y Yucatán, con base en las colectas siguientes, las cuales fueron distribuidas bajo varios nombres, como se cita a continuación: MEXICO, Tamaulipas: western slopes of the Sierra de Tamaulipas, at Santa María de los Nogales, elev. $800 \mathrm{~m}$, 23-IX-1956, F. Martínez Martínez \& G. Borja Luyando F-2112 (TEX), Adelia barbinervis Schlecht.; Mpio. de Aldama, Sierra de Tamaulipas, Rancho Las Yucas, 9-X-1957, R.L. Dressler 2319 (MEXU), Adelia barbinervis; $1 \mathrm{mi}$ W of El Abra, 28-X-1959, M.C. Johnston \& J. Graham 4559 (MEXU, TEX), Adelia barbinervis ?; Mpio. Gómez Farías, $6 \mathrm{~km}$ al SE de G. Farías, IX-1972, F. G. Medrano 4862 (MEXU), Phyllanthus nobilis (L.) Muell. Arg. San Luis Potosí: Mpio. Cd. Valles, Rancho Tinaja, 22-V-1981, P. A. Fryxell \& W. R. Anderson 3541 (ENCB, MEXU), det. correctamente por D. M. Johnson, 1982. Veracruz: Trapiche del Rosario, Actopan 28-X-1972, J. Dorantes et al. 01827 (XAL), Gymnanthes sp.; Plan del Río, E. Zapata, 14-I-1973, R. Hernández M. \& J. Dorantes 01823 (MEXU), Sebastiana sp. Yucatán: borde de la leguada Xnobekan, al SSE de Becanchen, 30-I-1956, O. G. Enríquez 382 (MEXU), Sebastiana n.?

Otros ejemplares examinados y determinados por la presente autora son: MEXICO, Jalisco, Mpio. La Huerta: 33.4 millas de Chamela, adelante del Río San Nicolás, 12-XII1970, L.A. Pérez J. 380 (MEXU); Estación de Biología Chamela, 5-VIII-1983, E. J. Lott 1778 (MEXU), 13-XII-1983, E. J. Lott 2181 (MEXU); Cerro Colorado, a ca $2 \mathrm{~km}$ al SE de la Estación de Biología Chamela sobre la carretera Puerto Vallarta-Barra de Navidad, 4-XII-1982, E. J. Lott \& T. Wendt 1616 (MEXU); Puente a ca $4 \mathrm{~km}$ al SE de la Estación de Biología Chamela, carretera Puerto Vallarta-Barra de Navidad, 1-XI-1982, E. J. Lott 1544 (MEXU); Rancho El Paraíso, 2-XII-1976, J. A. Solís Magallanes 280 (MEXU); Fraccionamiento Pueblo Careyes, 26-VII-1978, L. A. Pérez J. 1805 (MEXU). Herbarios consultados: CHAPA, ENCB, MEXU, MICH, TEX-LL, XAL. 


\section{LITERATURA CONSULTADA}

Liogier, E. E. (Hermano Alain). 1953. Euphorbiaceae. En: Flora de Cuba 3:41. Habana, P. Fernández.

Little, E. L. JR., R. O. WOOdbury y F. H. Wadsworth. 1974. Trees of Puerto Rico and the Virgin Islands 3:428-429.

URban, I. 1905. Symbolae Antillanae 4:337.

EmIly J. LotT. Herbario Nacional, Instituto de Biología, Universidad Nacional Autónoma de México, Apdo. Postal 70-367 04510 México, D.F. 